\title{
Taxonomic novelties in South American species of GutierRezia (Asteraceae, Astereae)
}

\author{
FRANCISCO RATTO ${ }^{1,2}$ and ADRIANA BARTOLI ${ }^{1,3}$
}

\begin{abstract}
Summary: During the revision of South American species of Gutierrezia (Asteraceae, Astereae) taxonomic novelties were established: (1) two new varieties were described, Gutierrezia mandonii var. anomala from northwestern Argentina, and Gutierrezia isernii var. nivea from central and western Argentina; and (2) the species Gutierrezia pulviniformis was included as a new synonym of Gutierrezia spathulata.
\end{abstract}

Key words: Argentina, Compositae, varieties, synonym.

\begin{abstract}
Resumen: Novedades taxonómicas en especies sudamericanas de Gutierrezia (Asteraceae, Astereae). Durante la revisión de las especies sudamericanas de Gutierrezia (Asteraceae, Astereae) se establecieron novedades taxonómicas: (1) Dos nuevas variedades fueron descriptas, Gutierrezia mandonii var. anomala del noroeste de Argentina y Gutierrezia isernii var. nivea del centro y oeste de Argentina; y (2) la especie Gutierrezia pulviniformis se incluyó como un nuevo sinónimo de Gutierrezia spathulata.
\end{abstract}

Palabras clave: Argentina, Compositae, variedades, sinónimo.

\section{INTRODUCTION}

Gutierrezia Lag. is a genus from the Americas, with a disjunct distribution in North and South America. In North America, 18 species inhabit in xerophytic or halophytic areas in the Central-West of the United States and South of Mexico (Nesom, 2006). On the other hand, 16 species occur in southern South America from Bolivia to extreme southern Argentina and Chile. Particularly, two species grow in Bolivia (Solbrig, 1966; Hind, 2011; Beck et al., 2014), six in Chile from the region I (Tarapacá) to the XII (Magallanes) (Zuloaga et al., 2008), and 12 in Argentina, mainly on the West from the border with Bolivia up to Tierra del Fuego archipelago (Zuloaga et al., 1999; 2008; Sancho \& Viera Barreto, 2014). Recently, three new species were described from

${ }^{1}$ Cátedra de Botánica Sistemática, Facultad de Agronomía, Universidad de Buenos Aires, Av. San Martín 4453, C1417DSE Buenos Aires, Argentina

${ }^{2}$ ratto@agro.uba.ar (author for correspondence)

${ }^{3}$ cbartoli@agro.uba.ar
Argentina (Ratto \& Bartoli, 2014, 2016a, 2016b), and two were resurrected (Ratto \& Bartoli, 2015, 2016c), bringing thus to 11 the Argentine species, nine of which are endemic.

In this work, taxonomic and nomenclatural novelties are presented which were found during the course of the revision of the genus Gutierrezia. Firstly, two new varieties of Gutierrezia are here described. On the other hand, the name $G$. pulviniformis is here proposed as a synonym of $G$. spathulata.

\section{Material and Methods}

The specimens of herbarium deposited in the following institutions were studied: BA, BAA, BAB, COL, CORD, F, G, GH, GOET, K, LP, MCNS, MERL, MPU, NY, P, SI, SGO and US. When physical specimens were not available, their photographs were obtained from JSTOR (plants.jstor.org) virtual herbaria or digital images were requested from curators of the herbaria and then analyzed. All the protologues 
were cross-checked with the label information of the type materials. A field trip was conducted in February 2016 with the objective of studying the populations of these varieties in their natural environment.

\section{Results}

A new variety of Gutierrezia mandonii

1. Gutierrezia mandonii (Sch. Bip.) Solbrig, Contrib. Gray Herb. Harvard Univ. 197: 20. 1966. 三 Brachyris mandonii Sch. Bip., Linnaea 34: 534. 1866. Type: Bolivia. La Paz, Larecaja, "vicinis Sorata, $2650 \mathrm{~m}$, II/III-1859, Mandon 228 (Holotype P 00742784 [photo!], isotypes COL 000004818 [photo!], F 0049093F [photo!], F 0049092F [photo!], G 00223900 [photo!], GH 00004315 [photo!], GH 00004316 [photo!], GH 00004317 [photo!], GOET 001597 [photo!], GOET 001598 [photo!], K 000221361 [photo!], MPU 023716 [photo!], MPU 023717 [photo!], MPU 023718 [photo!], NY 00162730 [photo!], NY 00162731[photo!], NY 00162732 [photo!], P 00742783 [photo!], P 00742786 [photo!], SI 014008 [photo!], US 00127684 [photo!]).

$=$ Gutierrezia gilliesii Griseb. var. scabriuscula Griseb., Abh. Königl. Ges. Wiss. Göttingen 19: 173. 1874. Type: Argentina. Catamarca, Nacimientos, Sierra de Catamarca, I-1872, Lorentz 434 (Holotype GOET 001593 [photo!], isotypes CORD 00006303!, LP 001995!, LP 001996!).

Subshrubs 5-25(-35) cm high, resinose, branched from the base. Stems numerous, decumbent, ribbed, glandular, leafy at the base, loosely leafy at apex. Sessile leaves erect, linearelliptic, 5-15 x 1-1.5 mm, with stipitate glandular trichomes and sessile glandular trichomes in pits on both blade surfaces, acute or subobtuse, subcoriaceus. Heads radiate, heterogamous, sessile, 1-1.5 cm diam., arranged in corymbiform cymes at stem apex, with 1 or 2 linear-elliptic bracts 1-5 $\mathrm{mm}$ de long. Involucre campanulate, resinose, 4-7 x 4-10 $\mathrm{mm}$. Receptacle flat or slightly convex, epaleate, pitted, pilose. Ray florets 8-15, pistillate, with ligule elliptic, 6-9 $\mathrm{mm}$ long. Disc florets 10-20, perfect, with corolla tubular, 3-4 mm long, throat abruptely ampliate. Anthers rounded at the base, apex of connective ovate-triangular, $0.5 \mathrm{~mm}$ long, style branches elliptic, acute, with sweeping papillose trichomes on the outer side. Cypselae terete-ovoid, 2-2.5 $\mathrm{mm}$, ribs densely sericeous, gray. Ray florets pappus of 10-18 erose marginated scales, linearelliptic, 1-1.5 mm long, disc florets pappus 1-2 $\mathrm{mm}$ long.

1.a. Gutierrezia mandonii (Sch. Bip.) Solbrig var. mandonii

Ray and tubular flowers golden yellow.

Iconography. Cabrera, 1978: 161, fig. 71; Sancho \& Viera Barreto, 2014: 184.

Etymology: In honour of Gilbert Mandon (1799-1866) a French mining engineer and botanist.

Geographical distribution and habitat: It inhabits in northwestern Argentina in Jujuy, Salta, and Tucumán provinces, and Bolivia in La Paz department. This species grows on rocky slopes, between 1500 and $3900 \mathrm{~m}$ elevation, in stony, sandy soil associated with grasslands and xerophytic shrubs such as species of Junellia Moldenke, Chuquiraga Juss. and Senecio L.

Phenology: It blooms in February.

1.b. Gutierrezia mandonii (Sch. Bip.) Solbrig. var. anomala Ratto \& A. Bartoli, nov. var. Type: Argentina. Salta, Chicoana, Cuesta del Obispo, La Herradura, S $25^{\circ} 10,909^{\prime}$, W $65^{\circ} 51,040^{\prime}, 3091 \mathrm{~m}, 19-\mathrm{II}-2016$, Ratto et al. 121/2016 (Holotype BAA 00004816!, isotypes BAA 00004817!, BAB!, BC!, CTES!, SI!). Fig. 1.

It differs from the typical variety by their heads with yellowish, whitish or rarely pale pink corollas.

Etymology. Name "anomala" refers to pink pale ray corollas that are uncommon in this species.

Geographical distribution and habitat: It inhabits northwestern Argentina, in Salta province, Chicoana department. It occurs in the transition between the phytogeographic provinces of Yungas and Prepuna. 


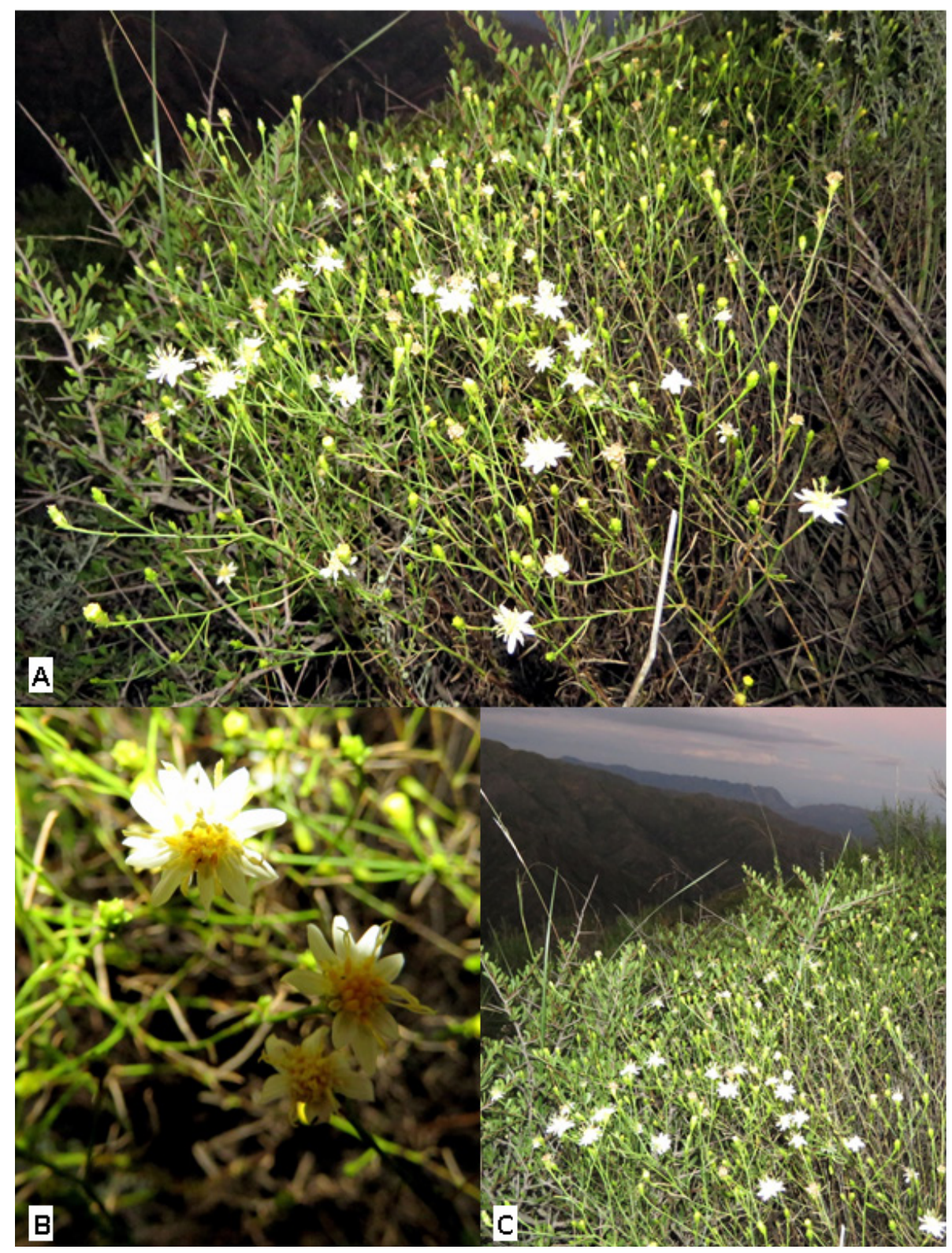

Fig. 1. Gutierrezia mandonii var. anomala A. Plant. B. Head. C. Habitat. Photos: F. Ratto.

Phenology: It blooms in February.

Additional material examined. ARGENTINA. Salta Prov.: Chicoana Dpt., Cuesta del Obispo, 3022 m, 20-II-2016, Ratto et al. 124/2016 (BAA); Piedra del Molino, 3360 m, 24-I-1995, Zuloaga et al. 11251 (SI); Parque Nacional Los Cardones, $2730 \mathrm{~m}, 14-\mathrm{II}-2002$, Cialdella et al.
268 (SI); Cuesta del Obispo, entre La Herradura y La Cochera, 3000 m, 2-IV-2004, Novara et al. 12063 (MCNS).

A new variety of Gutierrezia isernii

2. Gutierrezia isernii (Phil.) Phil., Anales Univ. Chile 87: 427. 1894. $\equiv$ Brachyris isernii Phil., 
Anales Univ. Chile 27: 337. 1865. Gutierrezia mandonii (Sch. Bip.) Solbrig subsp. isernii (Phil.) Solbrig, Contrib. Gray Herb. Harvard Univ. 197: 24. 1966. Type: Argentina. Mendoza, "inter Mendoza et Santa Rosa", 1861/63, Isern s.n. (Holotype SGO-65115!, isotype LP 001997!).

Shrubs $20-60 \mathrm{~cm}$ high, resinose. Stems erect, notably ribbed, glandular, leafy from the base. Sessile leaves, linear or linear-elliptic, 10-30 x 0.5-2 mm, apex acute, with stipitate and sessile glandular trichomes in pits on both blade surfaces, subcoriaceus. Heads on terminal branches, solitary, sessile, heterogamous, radiate, $8-10 \mathrm{~mm}$ diam., Involucre campanulate, $5-6 \times 2-4 \mathrm{~mm}$, terete, resinose, phyllaries in 3 series, graduated, oblong, with margin hyaline, outer phyllaries ovate, acute, apiculate, glandular; inner phyllaries triangular, acuminate glandular; bracts 1-2, linear o linear-elliptic, 1-5 mm long. Receptacle flat or slightly convex, epaleate, pitted, pilose. Ray florets 6-9, pistillate, with ligule elliptic, 5-6 mm long. Disc florets 6-10, perfect, with corolla tubular, 3-4 mm long, throat abruptly broadened. Anthers rounded at the base; apex of connective ovate-triangular, 0.5 $\mathrm{mm}$ long; style branches elliptic, acute or subobtuse with sweeping papillose trichomes on the outer side. Cypselae terete-ovoid, 1-1.5 mm, ribs densely sericeous, gray. Ray florets pappus of 10-15 linearelliptic scales $0.5-1 \mathrm{~mm}$ long, margin erose, disc florets pappus 1-2 $\mathrm{mm}$ long.

\section{2.a. Gutierrezia isernii (Phil.) Phil. var. isernii}

Ray and tubular yellow flowers.

Etymology. In honour of the Spanish botanist Juan Isern Batlló y Carrera (1821-1866).

Geographical distribution and habitat: It grows in Catamarca, Córdoba, La Rioja, Mendoza, San Juan, and San Luis provinces, on slopes with rocky soil, between 900 and 2700 m elevation.

Phenology: It blooms from November to July.

Common name: "yerba de sapo".

2.b. Gutierrezia isernii var. nivea Ratto \& A. Bartoli. nov var. Type: Argentina. Mendoza, Las Heras, Villavicencio, S $32^{\circ} 31,885^{\prime}$, W $69^{\circ} 01,328^{\prime}$, 2176 m, 15-II-2016, Ratto et al. 113/2016 (Holotype BAA 00004810!, isotypes BAA00004811!, BC!, SI!). Fig. 2.
It differs from the typical variety by its white, ray and disk florets.

Etymology: the name "nivea" refers to its white corollas.

Geographical distribution and habitat: It inhabits Córdoba (Santa María department), Mendoza (Godoy Cruz and Las Heras departments), and San Luis (La Capital department) provinces. It occurs in the phytogeographic province of Monte on slopes with stony soil, between 900 and $2700 \mathrm{~m}$ altitude.

Phenology: it blooms from January to July.

Additional material examined. ARGENTINA. Córdoba Prov.: Santa María Dpt., $3 \mathrm{~km}$ al S de San Pedro, 14-II-1962, Solbrig 3402 (LP). Mendoza Prov:: Godoy Cruz Dpt., Cerro Melocotón, 22-III1973, Roig 7705 (MERL). Las Heras Dpt., ca. del cerro Casa de Piedra, en el Mariño, 1350 m, 22VII-1975, Roig 8741 (MERL); hotel Villavicencio, 21-II-1977, without collector 97421 (SI); San Isidro, 1350 m, 12-IV-1979, Roig 9723 (MERL); Ruta 52, hotel Villavicencio, $1750 \mathrm{~m}, 11-\mathrm{I}-2011$, Ratto et al. 32, 33, 34, 35, 37, 95 (BAA). San Luis Prov.: La Capital Dpt., Sierra Varela, 22-XII-1925, Castellanos s.n. (BA); Potrero de Los Funes, 1189 m, 3-III-2014, Sbarra 20, 21, 22, 23, 24, 26 (BAA).

Gutierrezia pulviniformis as a new synonym of $G$. spathulata

G. pulviniformis was described by Cabrera (1940) as a dwarf shrub. This species was only collected at Cerro Nevado in Mendoza province. It coexists with G. spathulata (Phil.) Kurtz, a morphologically related species which differs by its small plant size and sessile heads. Specimens of G. pulviniformis were found in some Argentine herbaria from a single collection put together by Boelcke in 1974 . A detailed study of herbarium specimens and field populations have allowed us to observe intermediate traits: plant size and length of head peduncles. We conclude here that it is the same species and morphologic differences are due to the growth in different heights and environmental conditions. Therefore, G. pulviniformis is synonymised under G. spathulata in this paper.

3. Gutierrezia spathulata (Phil.) Kurtz, Bol. Acad. Nac. Ci. 13: 194. 1893. EBrachyris spathulata 


\section{F. Ratto and A. Bartoli -Taxonomic novelties in South American species of Gutierrezia}

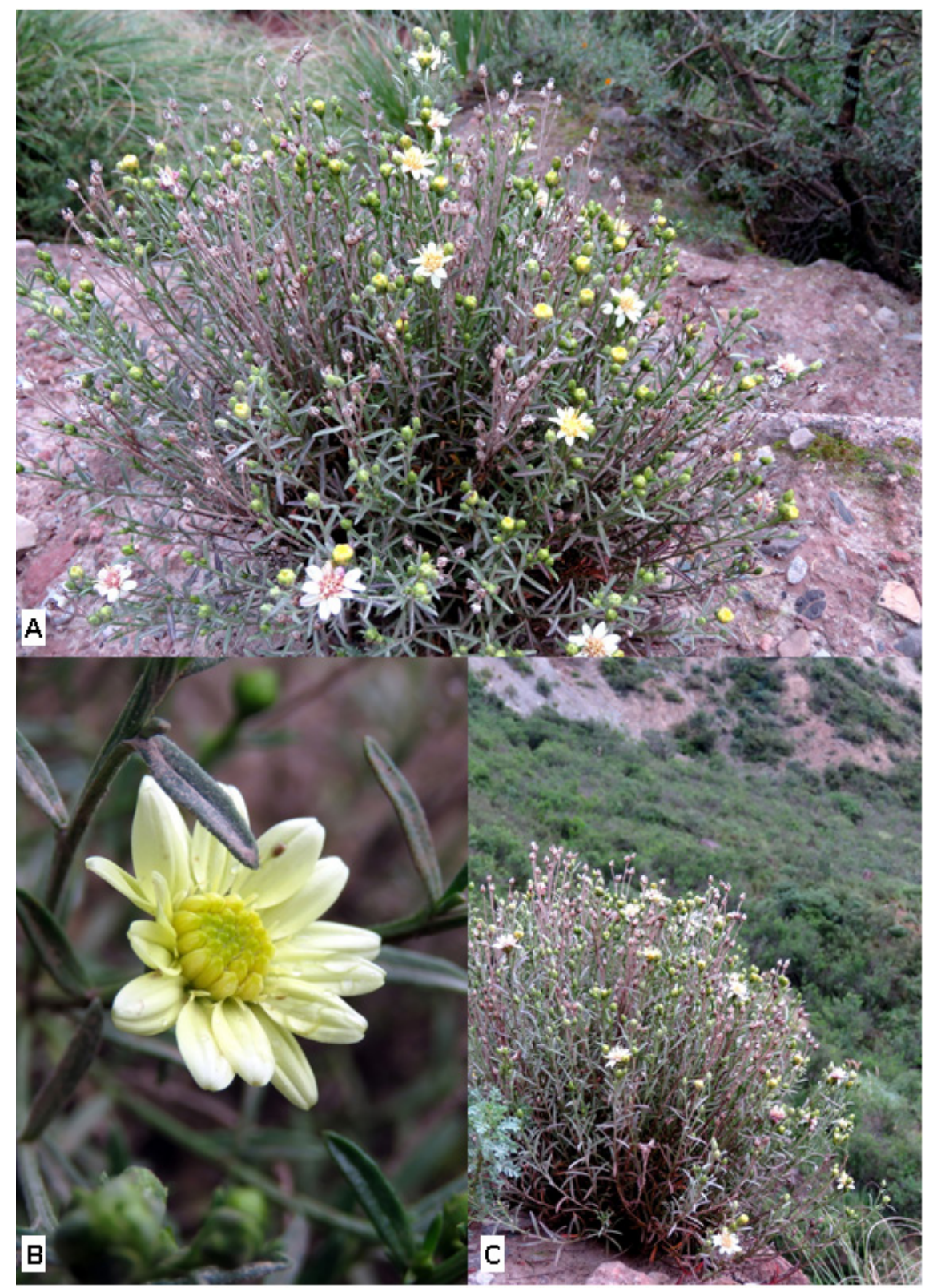

Fig. 2. Gutierrezia isernii var. nivea A. Plant. B. Head. C. Habitat. Photos: F. Ratto.

Phil., Anales Univ. Chile 27: 336. 1865. Type: Argentina. Mendoza, "prope la Guardia", Landbeck s.n. (Lectotype SGO-44787 [photo!], isotypes LP 002064!, SGO 65124 [photo!]), designated by Ratto (in press).

$=$ Gutierrezia spathulata (Phil.) Kurtz var ochroleuca Kurtz, Bol. Acad. Nac. Ci. 13: 186.
1893. Type: Argentina. Mendoza, "inter arroyo Papagayos et arroyo Hondo", 27-XII-1892, Kurtz 7431 (Holotype CORD 00005398! "Hoja A", CORD 00005399! "Hoja B", isotypes LP 002065!, LP 002066!).

= Gutierrezia pulviniformis Cabrera, Darwiniana 4: 135. 1940. Type: Argentina. Mendoza, San 
Rafael, Cerro Nevado, I-1916, Carette s.n. (Lectoype MERL [photo!], designated by Ratto et al. (in press); isolectotype LP 000272!).

Subshrubs 3-30(-40) cm high, resinose, branched at the base. Stems erect, when young ribbed, glandular, leafy at the base and loosely leafy to the apex. Sessile leaves elliptic-spatulate sessile, attenuated, pseudopetiolate, with stipitate trichomes and sessile trichomes in pits on both surfaces, subcoriaceous, spatulate basal or elliptic-spatulate $10-25$ x 2.5-6 mm, obtuse, caulinar leaves gradually smaller. Heads grouped in corymbiform cymes at the stems apex, sessile, heterogamous, radiate, 10-12 mm diam.,. Involucre ovoid, resinose, 4-6 x 3-5 mm. Receptacle flat or slightly convex, naked, pitted, pilose. Phyllaries in 3 graduated series, oblong, outer phyllaries glandular, acuminate, inner phyllaries triangular, glandular, apiculate, 1-2 linear-elliptic bracts 1-3 mm long. Receptacle flat or slightly convex, epaleated, pitted, pilose. Ray florets 5-7, pistillate, with ligule elliptic, 5-6 $\mathrm{mm}$ long. Disk florets 6-10, perfect, with corolla tubular, 3-4 $\mathrm{mm}$ long with an abruptely ampliate throat. Anthers with rounded at the base; apex of connective ovatetriangular $0.5 \mathrm{~mm}$. Style branches acute. Cypselae terete-ovoid, $1 \mathrm{~mm}$. long, ribbed, densely sericeous, gray. Pappus of ray florets 7-10 scales, linearelliptic, $0.5-1.5 \mathrm{~mm}$ long, margin erose, pappus in disk florets $0.5-1.5 \mathrm{~mm}$ long.

Iconography: Cabrera, 1971: 32, fig. 18; Sancho \& Viera Barreto, 2014: 186.

Etymology: name "spathulata" refers to the spatulate leaf shape.

Geographical distribution and habitat: It grows in Mendoza province and inhabits phytogeographical province of Patagonia, between 1300 and $2400 \mathrm{~m}$, in stony hillsides and crevices of rocks.

Phenology: It blooms from November to March.

Common names: "yerba de la oveja", "yerba del buitre", "yerba del guanaco".

Additional material examined. ARGENTINA. Mendoza Prov.: Maipú Dpt., Beltrán, Valle del Malargüe, 5-I-1988, Kurtz 5710, 5711, 5775, 7189 (CORD); Beltrán, La Cienaguita, Río Salado, 25-I-1982, Kurtz 7097 (CORD). Malargüe Dpt., Portezuelo del Choique, 2400 m, 12-III-1904, Ruiz Leal 16079 (MERL); Portimalal, 1700 m, 28-I-
1941, Ruiz Leal 7484 (MERL); La Valenciana, 2100 m, 4-II-1942, Ruiz Leal 7815 (MERL); La Valenciana, 4-II-1942, Ruiz Leal 7815 (BA); cerros al S de Calmuco, 15-XI-1942, Burkart et al. 14386 (SI); Portezuelo del Viento, 29-XII-1944, Ruiz Leal 9736 (MERL); Portezuelo del Viento, 29-XI-1944, Ruiz Leal 9735 (MERL); Los Colgados, 8-III-1954, Ruiz Leal 16015 (MERL); Valle del Atuel, 1920 m, 25-I-1964, Ruiz Leal 23166 (MERL); Camino a Los Molles, 8-XII-1970, Sosa 28772 (MERL); Los Molles, 8-XII-1970, Without collector (SI 138309); Sierra del Nevado, arroyo Chacay-co, puesto Barroso, $1300 \mathrm{~m}$, 3-XII-1973, Boelcke et al. 15566 (MERL); Sierra del Nevado, Cerros del Agua de la India Muerta, 10-XII-1975, Boelcke et al. 15792 (MERL); Los Molles, 26-XI-1977, Cordo 77-d-9 (SI); Los Molles, 26-XII-1977, Cordo 77-d10 (SI); Cerro Los Menucos, 2300 m, 18-XII-1980, Méndez \& Wuilloud s.n. (MERL); 5-6 km al N de El Manzano, 1700 m, 25-III-1980, Méndez \& Wuilloud s.n. (MERL); ladera $\mathrm{S}$ de cono volcánico, $1610 \mathrm{~m}$, 25-III-1980, Méndez \& Wuilloud s.n. (MERL); meseta de la Sierra de la Media Luna, 2300 m, 22I-1981, Del Vitto et al. 31488 (MERL); próximo al Volcán Dolo, 23-II-1984, Dalmasso 325 (MERL); Valle del Salado, 1750 m, 22-XII-1985, Lagiglia 6870 (SI); acceso Mina Ethel, 9-XII-1994, Lagiglia 8754 (SI); Sierras de Chachahuen, 20-XI-2001, Prina \& Escudero 1205 (SI); Sierras de Chachahuen, 12-XII-2001, Prina \& Troiani 1435 (SI); Reserva Provincial La Payunia, 1703 m, 27-XI-2002, Prina et al. 1788 (SI); Bordo Alto del Payún, 1700 m, 24XI-2004, Prina et al. 2568 (SI); Mina Ethel, 1700 m, 23-XI-2004, Prina et al. 2554 (SI); Ruta Provincial 180, entre Pampa de los Pajaritos y Mina Ethel, 1805 m, 10-XII-2004, Prina et al. 2590 (SI); Paso Pehuenche, $1760 \mathrm{~m}, 24-\mathrm{XI}-2010$, Zuloaga et al. 12449 (SI). San Carlos Dpt., 1500 m, 26-III-1918, without collector (LP 60534). San Rafael Dpt., 29XI-1920, Ruiz Leal 9735 (LP); Portimalal, 1700 m, 28-I-1941, without collector (LP 60373); entre Chihuido y Bardas Blancas, 12-II-1942, Covas 405, 406 (LP); Sierra del Nevado, arroyo Chacay-co, 1300 m, 3-XII-1973, Boelcke et al. 1566 (BAA); Cerro Nevado, alrededor de la Cienaguita, $2350 \mathrm{~m}$, 8-XII-1973, Boelcke et al. 15695 (BAB); Sierra del Nevado, Cerros de Agua de la India Muerta, 1750 m, 10-XI-1973, Boelcke et al. 15792 (BAB); Sierra del Nevado, $1.5 \mathrm{~km}$ al W de La Cienaguita, 22-I-1974, Boelcke et al. 15897 (BAB); Sierra del Nevado, 


\section{F. Ratto and A. Bartoli -Taxonomic novelties in South American species of Gutierrezia}

entre la Cienaguita y el Zanjón del Plateado, 24I-1974, Boelcke et al. 15968 (BAB); Sierra del Nevado, 2500 m, 22-I-1974, Bolecke et al. 15913 (BAA, BAB, MERL, SI); Cuadro Benegas, $1800 \mathrm{~m}$, 17-II-1982, without collector (MERL 32698); Ruta Provincial 184, 1386 m, 6-I-2015, Ratto et al. 30 (BAA); Cerro La Montura, 2206 m, 7-I-2015, Ratto et al. 52, 53 (BAA); El Sosneado, Ruta Provincial 220, 1930 m, 8-I-2015, Ratto et al. 77, 78, 79, 80 (BAA).

\section{Acknowledgments}

The authors gratefully acknowledge the support provided by the Universidad de Buenos Aires. We also thank T. Moloney who improved the English of this paper and two anonymous reviewers for their constructive comments, which helped us to improve the manuscript.

\section{Bibliography}

BECK, S.G., D. IBAÑEZ, P. M. JØRGENSEN, J. MÜLLER, J. F. PRUSKI, H. ROBINSON, E. URTUBEY, K. TREMETSBERGER \& T. STUESSY. 2014. Asteraceae. In: JØRGENSEN, P. M., M. H. NEE \& S. G. BECK (eds.), Catálogo de las plantas vasculares de Bolivia. Monogr. Syst. Bot. Missouri Bot. Gard. 127: 290-382.

CABRERA, A. L. 1940. Cuatro compuestas nuevas de la América Austral. Darwiniana 4: 133-140.

CABRERA, A.L. 1971. CABRERA, A. L. 1971. Compositae. In: CORREA, M. N. (ed.), Flora Patagónica. Tomo 8, Parte 7, pp. 1-451. Instituto Nacional de Tecnología Agropecuaria, Buenos Aires.

CABRERA, A. L. 1978. Compositae. In: CABRERA, A. L. (ed.), Flora de la Provincia de Jujuy, República Argentina. Tomo 13, Parte 10, pp. 1-726. Instituto Nacional de Tecnología Agropecuaria, Buenos Aires.

HIND, D. J. N. 2011. An annotated preliminary checklist of the Compositae of Bolivia (version 2) Available from: http://www.tropicos.org/ (Access: 7 April 2017).

LAGASCA, M. 1816. Gutierrezia. Genera et species plantarum, quae aut novae sunt aut nondum recte cognoscuntur. Matriti: ex Typographya Regia. Madrid.

NESOM, G. L. 2006. Gutierrezia. In: Flora of North America Editorial Committee (ed.). Flora of North America \& North of Mexico, 20: 88-94. Oxford University Press, Oxford.
PHILIPPI, R. A. 1865. Excursión botánica en Valdivia desde los Cuneos en el departamento de La Unión, a través de la Cordillera de la Costa, hasta el mar. Anales de la Universidad de Chile (Santiago) 27: 289-351.

RATTO, F. \& A. BARTOLI. 2014. Gutierrezia mendocina (Asteraceae, Astereae), a new South American species. Collectanea Botanica 33: e002. doi: http://dx.doi.org/10.3989/collectbot.2013. v33.002

RATTO, F. \& A. BARTOLI. 2015. The resurrection of Gutierrezia ameghinoi Speg. (Asteraceae, Astereae, Solidagininae), a species from Patagonia, Argentina. Phytotaxa 220: 295-300.

RATTO, F. \& A. BARTOLI. 2016a. A new species of Gutierrezia (Asteraceae, Astereae) from Argentina. Collectanea Botanica 35. http://dx.doi.org/10.3989/ collectbot.2016.v35.004.

RATTO, F. \& A. BARTOLI. 2016b. A new species of Gutierrezia (Asteraceae, Astereae, Solidagininae) from Argentinian Patagonia. Phytotaxa 266: 157-160.

RATTO, F. \& A. BARTOLI. 2016c. Reinstatement of Gutierrezia leucantha Cabrera (Asteraceae, Astereae). Webbia. DOI: 10.1080/00837792.2016.1180756.

RATTO, F., D. SCHIAVINATO \& A. BARTOLI. In press. Typification of names in South American Gutierrezia (Asteraceae, Astereae). Collectanea Botanica.

SANCHO, G. \& J. N. VIERA BARRETO. 2014. Gutierrezia Lag. In: ZULOAGA, F. O., M. J. BELGRANO, \& A. M. ANTON (eds.), Flora Argentina: Flora vascular de la República Argentina, vol. 7, t. 1, pp. 181-187. Estudio Sigma SRL, Buenos Aires.

SCHULTZ BIPONTINUS, C. H. 1866. Enumeratio Cassiniacearum a cl. G. Mandon in Bolivia a. 18571861 lectarum. Linnaea 34: 527-536.

SOLBRIG, O. 1966. The South American species of Gutierrezia. Contrib. Gray Herb. 197: 3-42.

ZULOAGA, F. O. \& O. MORRONE (eds.). 1999. Catálogo de Plantas Vasculares de la República Argentina II, . Achanthaceae-Euphorbiaceae. Monogr. Syst. Bot. Missouri Bot. Gard. 74: 1-621.

ZULOAGA, F. O, O. MORRONE \& M. J. BELGRANO (eds.). 2008. Catálogo de las Plantas Vasculares del Cono Sur (Argentina, Sur de Brasil, Chile, Paraguay y Uruguay). Monogr. Syst. Bot. Missouri Bot. Gard. 107(2): 985-2286.

Recibido el 10 de febrero de 2017, aceptado el 23 de mayo de 2017. 
